\title{
To study the clinical, radiological and functional outcome of antibiotic mixed cement coated nails in infected long bone diaphyseal fractures
}

\author{
Uikey S. ${ }^{1}$, Oddam V.K. ${ }^{2}$, Verma R. ${ }^{3}$ \\ ${ }^{1}$ Dr. Suresh Uikey, Assistant Professor, ${ }^{2}$ Dr. Vinay Kumar Oddam, Postgraduate Student, ${ }^{3}$ Dr. Rahul Verma, Associate \\ Professor; all authors are affiliated with Department of Orthopedics, Gandhi Medical College and Hamidia Hospital, Bhopal, \\ M. P., India
}

Corresponding Author: Dr. Vinay Kumar Oddam, Email: dr.vinayoddam@gmail.com

\begin{abstract}
Background: In Present Era, High velocity trauma are common trends leading to compound long bone fractures. These fractures have high chance of infection which further leads to infected non-union. Traditionally multistage procedure such as debridement and systemic antibiotics with temporary fixation and then permanent fixation and bone grafting when required. Single staged procedures such as debridement and application of Ilizarov fixator and limb reconstruction system are also available. Recently, development ofinfection control by local antibiotic delivery systems, initially antibiotic cement beads then antibiotic cement impregnated intramedullary nail (ACIINs). The aim of this study is to analyze clinical, functional and radiological outcome of antibiotic mixed cement coated nailing in infected long bone diaphyseal fractures. Material and Methods: In our prospective study, 20 patients of 17-70 years of age group with infected long bone diaphyseal fractures were treated using antibiotic mixed cement coated intramedullary nails. The outcome of study was analyzed for infection control, radiological sign of union, ability to bear weight and patient compliance. Results: In our study, we achieved infection control in $95 \%$ of patients with complete radiological union in $50 \%$ of cases with ability to bear weight without pain. Conclusion: Antibiotic Mixed Cement Coated Nailing was a good procedure to achieve infection control, provide stability and bone union in infected long bone fractures.
\end{abstract}

Key words: velocity trauma, ACIINs, Ilizarov fixator.

\section{Introduction}

Long bone fractures are debilitating injuries commonly resulting from high-energy trauma. Mechanization of mobility, rapid industrialization and urbanization has led to a tremendous increase in the density of vehicular traffic, which makes automobile accidents an inevitable event. High velocity trauma is leading to increased and varying types of injuries.

The problems include large soft tissue defects along with exposed fractured bone fragments. Successful managements of open fractures of leg depend upon both the structural integrity of skeletal fixation and viable soft tissue coverage. Even the closed fracture of long bone posses' problem and the final outcome is doubtful and when this is superadded by wound, the problem of management are increased manifold.Management of compound fracture of long bones includes two principles:(a) Management of soft tissue injuries and

Manuscript Received: $12^{\text {th }}$ December 2017

Reviewed: $22^{\text {nd }}$ December 2017

Author Corrected: $20^{\text {th }}$ December 2017

Accepted for Publication: $26^{\text {th }}$ December 2017 prevent infection. (b) Management of fracture by proper skeletal stabilization. Both of these are complimentary to each other and result of one directly affects the outcome of other. The main goal in management of these injuries is conversion of open fracture into closed fracture and stabilization and immobilization of fracture to achieve a sound bony union. If any lag in management of these component, may lead to infected fracture, and treating infected fracture of bone is a real challenge.Infected long bone fracture is a debilitating disorder that still poses a very complex problem to the surgeon today in term of cost and long duration of treatment [1].

Post-traumatic infection occurred in as many as $10 \%$ of all open fractures and $1 \%$ of all closed fractures [2]. Causes of infected long bone fracture are generally inherent to the fractures, like compound fracture, loss of soft tissue or bone etc [3]. The risk of infection following IM nailing of closed long bone fractures is thought to be similar to the general risk of infection after any orthopedic trauma procedure, but this risk is substantially increased 


\section{Original Research Article}

in the setting of open fractures and has been reported to range between $4 \%$ and $7 \%$ [4]. A prevalence of methicillin-resistant or gentamicin-resistant Staphylococcus aureus (SA) has been observed, and bone infection with methicillin-resistant SA (MRSA) has increased among patients with implanted orthopedic devices [5]. Traditionally treatment of infected long bone fracture follows the multistage procedure such as debridement and systemic antibiotics with temporary fixation and then permanent fixation and bone grafting when required or bone transport $[3,6]$. For the treatment of infected long bone fracture Single staged procedures such as debridement and application of Ilizarov fixator and limb reconstruction system (LRS) are also available but these procedures are technically demanding, costly, require long term follow up, low patient compliance and have significant complications. These procedures are best suited for large segmental bone defect and both these procedures do not address to the infection control. This led to development ofinfection control by local antibiotic delivery systems, initially antibiotic cement beads then antibiotic cement impregnated intramedullary nail (ACIINs). The antibiotics cement beads control infection and fill dead space but do not provide any mechanical stability. Also require another procedure to remove and at time difficult to remove. Antibiotic cement impregnated intramedullary nail (ACIINs) provides a high concentration of antibiotics locally, fills dead space, gives good mechanical stability at fracture site and there by promotes bone healing[7,8]. Thus, Antibiotic cement impregnated intramedullary nail (ACIINs) provides all advantages of cement beads[9].

The present study was undertaken to analyze the usefulness of antibiotic cement impregnated intramedullary nail in case of infected fracture of long bones.

\section{Material and Methods}

This non-randomized prospective study in 20 patients presented at Department of Orthopedics and Traumatology, Gandhi Medical College and Hamidia Hospital, Bhopal, between October 2015-September 2017, with infected long bone diaphyseal fractures. Among 20, there were 19 males and 1 female, with the mean age being 34.25 years (range, 20-55 years).

In this study, we treated 10 tibias and 9 femurs and 1 humerus, with 19 patients having sustained high-velocity trauma and one patient having sustained domestic injury. 8 patients had intramedullary nail in situ, 8 patients had external fixator, and one patient had plate in situ when they presented to us and they were treated in different centers earlier. 12 patients had wound and 8 patients had pus discharging sinus. All patients when presented to us, had full blown infection. The mean duration between injury to index surgery was 9.8 days and mean duration between index surgery to antibiotic mixed cement coated nailing was 190.9 days.

Procedure: Before surgery a careful preoperative planning, pre-operative culture and sensitivity if possible and assessment of patient were done. All patients were subjected for complete pre-operative evaluation including the following investigations: complete blood count, ESR, C Reactive Protein, culture and sensitivity, sinogram. X-rays of the part in two views were also obtained. Regional block or general anesthesia was given after shifting the patient to operation theatre.

\section{Surgical Steps}

Step I: Radical debridement of wound and sinus was done by opening the Fracture site and wound with excision of infected bone ends and soft tissue. Medullary cavity washes open or close depending on size and location of wound or discharging sinus.

Step II: Preparation of canal- Medullary canal is reamed with reamers of increasing size so as to debride it completely and Reaming material was sent to culture sensitivity test. Then thorough wash with normal saline solution, and betadine solution with help of Foleys catheter was done and $50 \mathrm{ml}$ syringe.

Step III: Preparation of nail - In our series K-nail/V-nail/locking intramedullary nail was used for femur, tibia and humerus. The nail is prepared on a separate sterile table. Nail length is measured preoperatively. Nail diameter is determined by the largest reaming diameter. Kuntscher nail or V nail or regular intramedullary interlocking nail or Ender nail of $8 \mathrm{~mm}$ diameter is selected. Standard viscosity bone cement was used (40 gm). The cement was thoroughly mixed with antibiotics, following which the polymer was added. When the cement reaches doughy consistency, the selected nail is coated with bone cement using an endotracheal tube to make the diameter uniform along the length of the nail. In case intramedullary interlocking nails, screw holes are left uncemented to accommodate locking bolts. The diameter is checked with Kuntscher diameter 


\section{Original Research Article}

measuring gauge, excess cement is shaved off and the nail rerolled before the cement sets. The diameter is re checked. Bone cement is allowed to set for 15 minutes before insertion to allow the monomer to evaporate and to prevent cement nail debonding. In case of tibia proximal end of the nail was bending by nail bender like Herzog bend of tibia interlocking nail.

In our study Antibiotics we used $4 \mathrm{gm}$ of vancomycin powder and $3 \mathrm{gm}$ powder of Cefuroxime sodium and $2 \mathrm{gm}$ of Meropenem and mixed with 40 gm PMMA bone cement in a bowl and then resulting paste is coated to nail sparing the nail eye, then nail put for some time for setting of bone cement. All efforts are done to make the nail surface uniformly smooth. Now nail is ready to insert.

Step IV: Insertion of nail-Both ante-grade and retro-grade insertion of nail is performed for femur and ante-grade for tibia cases. Both proximal and distal locking done by free hand technique in case locking intramedullary nail was used. The wound will be again washed thoroughly and closure will be performed if possible. In case of soft tissue defect, flap cover with skin grafting done either in the same setting or later. In cases with bone loss bone grafting was required after infection control. The proximal and distal locking was done by free hand technique to provide stability. Dressing done and patient sifted out from OT.

Stage V: Postoperative management done as per routine protocol. Routine Antibiotic continues till culture sensitivity report was come. After receiving culture report we start antibiotic accordingly. X-ray done post operatively. Check dress done on $3^{\text {rd }}$ post operative day. Stitches removal was done on $12^{\text {th }}$ day. If required skin grafting done on same sitting or later depending on wound. Then patients followed up in every 4 week or SOS till union or further surgical intervention has been done.

Table-1: Criteria for assessment of final result.

\begin{tabular}{|c|c|c|c|c|c|}
\hline S. No. & Variable & Excellent & Good & Fair & Poor \\
\hline 1 & Infection & Eradicated & Control & Control & Not control (septicemia) \\
\hline 2 & Sinus/ wound & Healed & Healed & Healing & Not heal \\
\hline 3 & Radiological union & +++ & ++ & + & - \\
\hline 4 & Weight bearing without pain & +++ & ++ & + & + \\
\hline
\end{tabular}

\section{Results}

Results were evaluated with regard to infection control, radiological sign of union and ability to bear weight without pain.In our series of 20 cases, comprise 10 tibias, 9 femurs and 1 humerus. $40 \%$ of cases presented with infected intramedullary implant in-situ and another $40 \%$ patient have external fixator ex-situ, 1 patient was with infected plate in situ. Mean age 34.25 years (20-55), 19 male and 1 female, 95\% cases from high velocity road traffic accident. $35 \%$ culture positive for mixed organisms, $30 \%$ staphylococcus aureus, $10 \%$ Klebsiella and group-D streptococci each and E. coli, Coagulase negative staphylococci in remaining cultures. The mean duration between injury to index surgery was 9.8 days and mean duration between index surgery to present surgery was 190.9 days
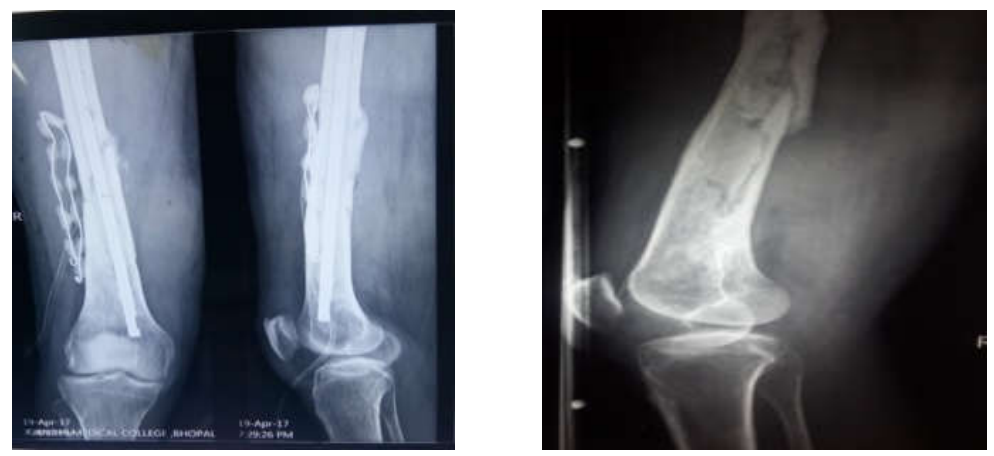

$10(50 \%)$ cases showed excellent result with eradication of infection, achieved boy union and able to bear weight without pain with good patient compliance.Good result in around $40 \%$ of fractures where infection controlled with some signs of union and patients were able to bear weight with some pain but there is need of secondary procedure like bone grafting to 


\section{Original Research Article}

achieve complete union. In around 5\% patients, showed fair result where delayed union present with persistent infection.In another 5\% patients, showed poor result where union not achieved with persistent infection. Infection control at the end of 3 week was $90 \%$ and 45 of cases require another procedure for bone healing. A total of 7 cases had complications. Shortening was found in 5 cases in which it was associated with persistent infection in 2 cases and with non-union in 1 case. Another patient had Implant broken and secondary infection in primary surgery while Foot drop was observed in 1 patient.

Table-2: Final assessment.

\begin{tabular}{|c|c|c|c|c|c|}
\hline S.N. & Bone & Excellent & Good & Fair & Poor \\
\hline 1 & Femur & $44.4 \%$ & $44.4 \%$ & 0 & $11.2 \%$ \\
\hline 2 & Tibia & $60 \%$ & $30 \%$ & $10 \%$ & 0 \\
\hline 3 & Humerus & 0 & $100 \%$ & 0 & 0 \\
\hline & Total & $\mathbf{5 0} \%$ & $\mathbf{4 0} \%$ & $\mathbf{5 \%}$ & $\mathbf{5 \%}$ \\
\hline
\end{tabular}
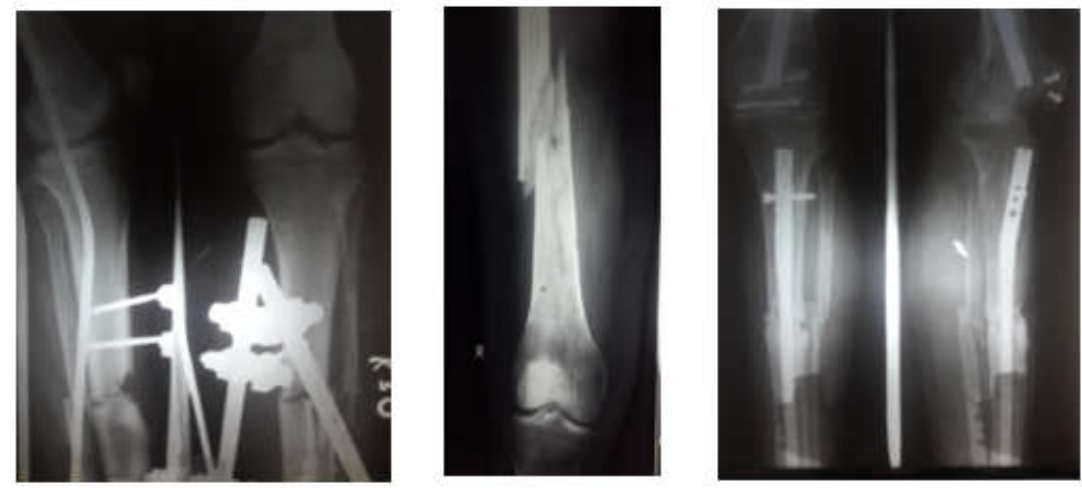

Figure 1: Radiological picture
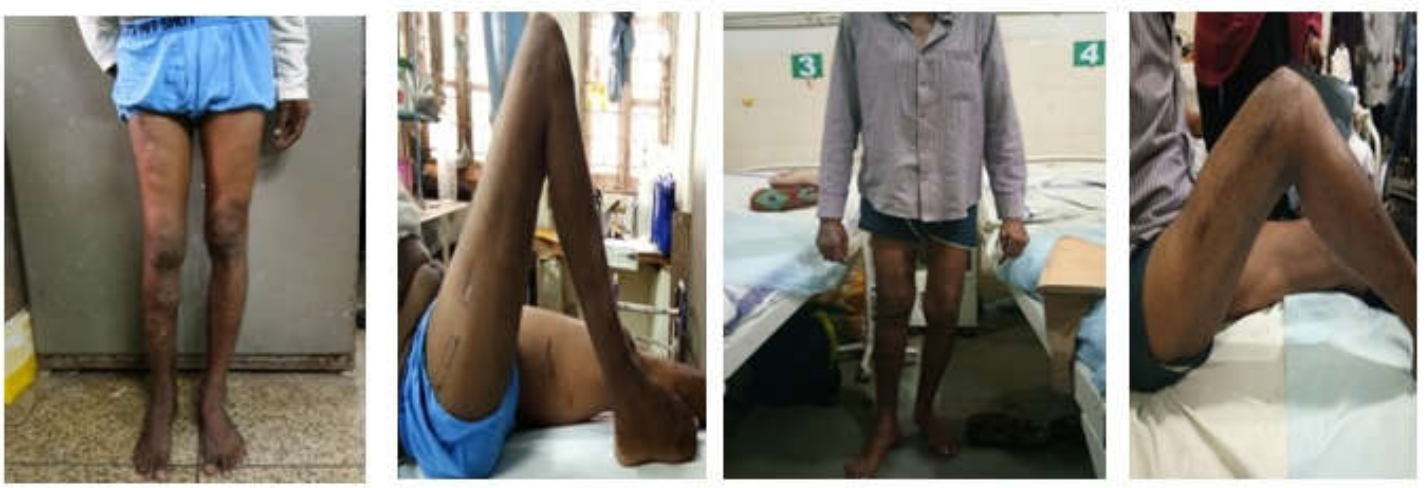

Figure 2:assessment of final result showing good improvement

\section{Discussion}

Infected long bone fractures require procedure to control infection, to provide stability, and to achieve union. Surgical debridement and delivery of antibiotic locally and systemically are used to control the infection [10].

Local antibiotic therapy results in high local concentration of antibiotic with minimum systemic level[10]. Use of antibiotic-impregnated cement was first noted by Buchholz and Engelbrecht in 1970.[11,12]. Antibiotic impregnated PMMA beads are used in treatment of osteomyelitis and open fracture [13]. However, a second procedure is requires removing the beads. Other method of local antibiotic delivery includes the use of bioabsorbable delivery vehicles such as calcium sulfate and synthetic polymers. New synthetic bio-absorbable delivery vehicle are being developed to avoid the need for a second procedure and in some case to promote osseous union [14-17].

Gentamicin and vancomycin are common choice for local delivery because of their wide spectrum activity and heat stability $[10,12,18,19]$. Bone cement eluted at locally 


\section{Original Research Article}

high concentration of antibiotic without systemic side effect. Antibiotic elusion from bone cement follows biphasic pattern with the initial rapid phase, followed by slow releasing phase after five to ten days. The safety of local antibiotic therapy is proven by clinical studies. Both animal and clinical studies show high concentration of antibiotic locally and undetectable or very low serum level of locally delivered antibiotic without systemic side effect.

Vancomycin, Meropenem and Cefuroxime are effective against many of the bacteria that were very commonly found in our patient. These antibiotics are heat stable with good local elution property from PMMA bone cement without effecting bone healing. In addition we give systemic antibiotic till culture and sensitivity report receives that was sent at the time of surgery, than antibiotic convert according to culture sensitivity report. These systemic antibiotics were continuing till patient admitted in hospital then shifted to oral antibiotic for six to eight weeks.

In our study Antibiotic mixed cement coated intramedullary nail control infection and provides stability in compound fracture and prevents development of chronic osteomyelitis and infected non union. In our series most common organism isolated was the staphylococcus aureus and mixed bacterial infection, which is also most common cause of osteomyelitis and infected non union.

Use of ACIIN has been first reported by Paley and Herzenberg [20] and later by other authors[7,8,21]. Paley et al have shown that control of infection was about $85 \%$ and bony union achieved in about $80 \%$ cases by ACIIN in infected non-union of long bones. Use of external fixators is associated with poor compliance and pin site complications. They are difficult to apply and maintain in obese patients. Such patients benefit from the antibiotic cement impregnated nailing.

Thonse and Conway in their study where infected nonunions were managed by antibiotic nails they achieved infection control in $85 \%$ of cases and union was seen in $84 \%$ cases. Around $27 \%$ patients required additional procedures for infection control and union. Most common complication in their series was cement debonding.

Our study differs from their study where in antibiotic cement interlocking nails were used. Infection control of our study $(92 \%)$ is comparable to their study $(85 \%)$.

Conflict of interest: None declared. Funding: Nil, Permission from IRB: Yes

\section{References}

1. Toh CL, Jupiter JB. The infected nonunion of the tibia. Clin Orthop Relat Res 1995;315:176-91

2. Patzakis MJ, Zalavras CG. Chronic posttraumatic osteomyelitis and infected nonunion of the tibia: current management concepts. J Am Acad Orthop Surg. 2005;13 (6): 417-27.

3. Court-Brown CM. Fractures of the tibia and fibula. In: Bucholz RW, Heckman JD, Court-Brown CM, editors. Rockwood and Green's fractures in adults. 6th ed. Lippincott Williams and Wilkins; 2006. p. 2080-146

4. Young S, Lie SA, Hallan G, Zirkle LG, Engesæter LB, Havelin LI. Risk Factors for Infection after 46,113 Intramedullary Nail Operations in Low- and Middleincome Countries. World J Surg. 2013; 37(2):349-55. [PMC free article][PubMed]

5. Gianluca Giavaresi, Veronica Borsari, Milena Fini, Roberto Giardino, Vittorio Sambri, Paolo Gaibani, Renzo Soffiatti. Preliminary Investigations on a New Gentamicin and Vancomycin-Coated PMMA Nail for the Treatment of Bone and Intramedullary Infections: An Experimental Study in the Rabbit.. DOI 10.1002/jor.20543

6. Ueng SW, Chuang DC, Cheng SL, Shih CH. Management of large infected tibial defects with radical debridement and staged double-rib composite free transfer. J Trauma 1996;40:345-9.

7. Qiang Z, Jun PZ, Jie XJ, Hang L, Bing LJ, Cai LF. Use of antibiotic cement rod to treat intramedullary infection after nailing: Preliminary study in 19 patients. Arch Orthop Trauma Surg 2007; 127:945-51.

8. Thonse R, Conway J. Antibiotic cement-coated interlocking nail for the treatment of infected non-unions and segmental bone defects. J Orthop Trauma 2007;21: 258-68

9. Rajesh dhanasekhar et al. Antibiotic cement impregnated nailing in management of infected non union of femur and tibia :Kerala J Orthop 2013; 26:93-97.

10. Zalavras CG, Patzakis MJ, Holtom P. Local antibiotic therapy in the treatment of open fractures and osteomyelitis. Clin Orthop Relat Res. 2004;427: 8693.

11. Wininger DA, Fass RJ. Antibiotic-impregnated cement and beads for orthopedic infections. Antimicrob Agents Chemother 1996;40:2675-9. 


\section{Original Research Article}

12. Buchholz HW, Elson RA, Heinert K. Antibioticloaded acrylic cement: Current concepts. Clin Orthop Relat Res 1984;190:96-108.

13. Henry SL, Ostermann PA, Seligson D. The prophylactic use of antibiotic impregnated beads in open fractures. J Trauma. 1990;30:1231-8.

14. Blaha JD. Calcium sulfate bone void filler. Orthopaedics 1998;21:1017-9

15. HanssenAD. Local antibiotic delivery vehicle in the treatment of musculoskeletal infection. Clin Orthop 1994; 298:229-39

16. Heijinkl A, Yeszemski MJ, Patel R, Rouse MS, Lewallen DG, Hanssen AD, Local antibiotic delivery with Osteoset,DBX,and Collagraft.Clin orthop 2006;451:29-33

17. Nelson CL, MacLaren SG, Skinner RA, SmeltzerMS, Thomas JR, Olsen KM. The treatment of experimental osteomyelitis by surgical debridement and the implantation of calcium sulfate tobramycin pellets. J Orthop Res 2002;20:643-7.

18. Springer BD, Lee GC, Osmon D, Haidukewych GJ, Hanssen AD, Jacofsky DJ. Systemic safety of high-dose antibiotic-loaded cement spacers after resection of an infected total knee arthroplasty. Clin Orthop Relat Res 2004;427:47-51.

19. Koo KH, Yang JW, Cho SH, Song HR, Park HB, Ha $\mathrm{YC}$, et al. Impregnation of vancomycin, gentamicin, and cefotaxime in a cement spacer for two-stage cementless reconstruction in infected total hip arthroplasty. J Arthroplasty 2001;16:882-92.

20. Paley D, Herzenberg JE. Intramedullary infections treated with antibiotic cement rods: Preliminary results in nine cases. J Orthop Trauma 2002;16:723-9.

21. Madanagopal SG, Seligson D, Roberts CS. The antibiotic cementnail for infection after tibial nailing. Orthopedics 2004;27:709-12.

\section{How to cite this article?}

Uikey S, Oddam V.K, Verma R. To study the clinical, radiological and functional outcome of antibiotic mixed cement coated nails in infected long bone diaphyseal fractures. Surgical Update: Int J surg Orthopedics.2017;3(4):169-174.doi:10. 17511/ijoso.2017.i04.13. 